\title{
Chitosan Hydrogel Micro-bio-devices with Complex Capillary Patterns via Reactive-Diffusive Self-Assembly
}

\author{
Vahid Adibnia ${ }^{\mathrm{a}}$, Marziye Mirbagheri ${ }^{\mathrm{a}, \mathrm{b}, \mathrm{c}}$, Pierre-Luc Latreille ${ }^{\mathrm{a}}$, Jimmy \\ Faivre $^{\mathrm{a}, \mathrm{d}}$, Bruno Cécyre ${ }^{\mathrm{e}}$, Jean-Francois Bouchard ${ }^{\mathrm{e}}$, Vincent A. Martinez ${ }^{\mathrm{f}}$, \\ Thierry Delair ${ }^{\mathrm{d}}$, Laurent David ${ }^{\mathrm{d}}$, Dae Kun Hwang ${ }^{\mathrm{b}, \mathrm{c}}$, Xavier Banquy ${ }^{\mathrm{a}, *}$ \\ ${ }^{a}$ Faculty of Pharmacy, Université de Montréal, Montreal, Quebec H3C 3J7, Canada \\ ${ }^{b}$ Department of Chemical Engineering, Ryerson University, Toronto, Ontario M5B 2 K3, \\ Canada \\ ${ }^{c}$ Keenan Research Center, Li Ki Shing Knowledge Institute, St. Michael's Hospital, \\ Toronto, Ontario M5B 1 W8, Canada \\ 'Ingénierie des Matériaux Polymères (IMP@Lyon1), CNRS UMR 5223, \\ Université de Lyon, Université Claude Bernard Lyon 1, \\ 15 Boulevard Latarjet, 69622 Villeurbanne Cedex, France \\ e School of Optometry, Université de Montréal, Montreal, Quebec, H3C 3J7, Canada \\ ${ }^{f}$ School of Physics and Astronomy, The University of Edinburgh, Edinburgh, EH9 3FD, UK
}

\begin{abstract}
We present chitosan hydrogel microfluidic devices with self-assembled complex microcapillary patterns, conveniently formed by a diffusion-reaction process. These patterns in chitosan hydrogels are formed by a single-step procedure involving diffusion of a gelation agent into the polymer solution inside a microfluidic channel. By changing the channel geometry, it is demonstrated how to control capillary length, trajectory and branching. Diffusion of nanoparticles (NPs) in the capillary network is used as a model to effectively mimic the transport of nano-objects in vascularized tissues. Gold NPs diffusion is measured locally in the hydrogel chips, and during their two-step transport through the capillaries to the gel matrix and eventually to embedded cell clusters in the gel. In addition, the quantitative analyses reported in this study provide novel opportunities for theoretical investigation of capillary formation and propagation during diffusive gelation of biopolymers.
\end{abstract}

Keywords: biomaterials, chitosan, self-assembly, tissue engineering, drug

\footnotetext{
* Corresponding author

Email address: xavier.banquy@umontreal.ca (Xavier Banquy)
} 
delivery, microfluidics

\section{Introduction}

Hydrogels are attractive soft materials for regenerative medicine mainly because of their ability to mimic the microstructure and various physical and biological properties of natural tissues. [1, 2] Ease of hydrogel production and 5 processing enables cell encapsulation and growth, 3, 4, as well as drug loading and controlled release. [5, 6] Hydrogels, regardless of their preparation technique (e.g., self-assembly, physical or chemical crosslinking), typically have isotropic microstructures, possibly heterogeneous with spatially-variant crosslinking densities. [7] Hydrogel micropatterning techniques have been used to improve the integration of the hydrogels in biological systems, to emulate the natural tissue environment, and to improve drug testing applications. 8] However, hydrogel spatial patterning is challenging and often requires a multi-step procedure. [9, 10]

Stereolithography (SLA) is the most commonly used technique for creating 15 patterned hydrogels, which involves photo-crosslinking of a printed pregel solution layer-by-layer, 11] or using a photomask preferentially under a microfluidic device. [12, 13] Despite the significant progress made in recent years on SLA and similar micropatterning techniques 9], self-assembled patterning has remained an important alternative for fabricating polymeric structures because of its simplicity, lack of toxic crosslinking compounds, and potentially quick and one-step production procedure. Nevertheless, controlling self-assembled patterns to obtain a desired hydrogel microstructure could be tedious, and only recently a few examples have emerged in the literature. [14, 15, 16]

Self-assembled anisotropic capillaries in hydrogels can be formed during 25 the physical crosslinking (gelation) of biopolymers, such as alginate, 17] collagen 18 and chitosan, 19 by diffusion of a gelation agent. These capillaries are typically a few millimeters to centimeters long and a few tens of micrometers in diameter. The mechanism of capillary formation in these biopolymer 
hydrogels has been studied extensively. Kohler and coworkers attributed the capillary formation to a Rayleigh-Benard-like instability, [20, 21, 22] whereas several detailed studies suggest that the process can be well explained by spinodal decomposition during the gelation process. [23, 18, 24] Regardless of the exact mechanism, capillary formation has always been found to depend on polymer nature and macromolecular structure, such as the molecular weight and monomer sequence, and gelation conditions, such as the polymer concentration, gelation agent (ion) type and concentration, and gelation geometry.

Among these gelable biopolymers, chitosan, a natural polysaccharide derived from chitin, is perhaps the most intriguing candidate in view of its physicochemical and structural properties. Unlike physically crosslinked calcium-alginate hy40 drogels that dissolve in aqueous solutions, physical hydrogels made of chitosan are stable in aqueous media at close-to-neutral $\mathrm{pH}$ values and in physiological environments. [25, 26, 27] The biodegradability, biodistribution and toxicity of chitosan have been studied extensively, confirming that chitosan is an excellent candidate for various biomedical applications. 25] Chitosan is soluble in mildly acidic solutions due to the protonation of its amine groups. Increasing pH decreases the polymer charge density, and eventually leads to gelation (if the molecular weight and degree of acetylation are within appropriate ranges [28]). The capillary formation during chitosan gelation is induced by one-dimensional (1D) diffusion of a strong base (usually $\mathrm{NaOH}$ ) through an acidic chitosan so50 lution. [19, 28, 29,

Although capillary formation in biopolymers has been studied for more than two decades, their practical applications have been reported by only a few studies. [30, 29, 31] The main issue that limits their practicality is that their formation is strictly controlled by the 1D diffusion of the gelation agent. To date, 55 no study has demonstrated any control over the shape and orientation of selfassembled capillaries in biopolymer hydrogels. In this study, we show that by producing the hydrogels in a microfluidic device, the 1D diffusion of the gelation agent could be indeed manipulated to generate programmed networks of capillaries. 
This study illustrates that, with a fine adjustment of the gelation agent diffusion in a microchannel, an unprecedented control over capillaries direction, branching, and repartition can be achieved. It will also be shown how these capillaries interact with obstacles in their pathway during formation. As a proof of concept, it will be then demonstrated how these "microvascularized" hydrogels can be used, for example, as a platform for simulating the diffusion of NPs in a tissue to reach targeted cellular areas, which is a complex process extensively investigated in drug delivery systems. 32 The unprecedented control over the chitosan hydrogels vascularization, in addition to their ease of fabrication, provide a powerful platform for nanoformulation screening and tissue engineering.

\section{Experimental Section}

\subsection{Chitosan preparation and characterization}

Deacetylated chitosan from squid pens was purchased from Mahtani Chitosan Pvt. Ltd. (Batch type 114, India). The acetylation and purification steps were followed as described previously to obtain a pure chitosan lyophilizate. 19,

75 29. The degree of acetylation (DA) was calculated from ${ }^{1} \mathrm{H}$ NMR spectroscopy (Bruker Avance III 400 US+ spectrometer, $400 \mathrm{MHz}$, [33, 19, 29] and the molar mass $\left(\mathrm{M}_{\mathrm{W}}\right)$ and dispersity $\left(\mathrm{M}_{\mathrm{W}} / \mathrm{M}_{\mathrm{n}}\right)$ were analyzed using multi-angle light scattering coupled with size exclusion chromatography (Wyatt Technology). The results are listed in Table 1.

Table 1: Chitosan macromolecular characteristics.

\begin{tabular}{ccc}
\hline $\mathrm{M}_{\mathrm{W}}(\mathrm{kDa})$ & $\mathrm{M}_{\mathrm{W}} / \mathrm{M}_{\mathrm{n}}$ & $\mathrm{DA}$ \\
\hline 604 & 1.64 & $4.3 \%$ \\
\hline
\end{tabular}

\subsection{Chitosan gelation}

Chitosan solutions were prepared by dispersing the purified chitosan powder in distilled water (mainly $1.5 \mathrm{wt} \%$ ), and adding stoichiometric amount of glacial 
acetic acid to ensure the complete protonation of the amine groups. The solution was mixed for at least $7 \mathrm{hrs}$ in a closed reactor for complete dissolution of chitosan. The resulted solution was then stored in refrigerator for $24 \mathrm{hrs}$ to remove air bubbles. The polymer solution was injected to gelation channels with different thicknesses (including the microfabricated channels) using a syringe. The $\mathrm{NaOH}$ solutions (mainly $1 \mathrm{M}$ ) for hydrogel preparation were prepared by mixing $\mathrm{NaOH}$ pellets (Sigma-Aldrich) in distilled water. The glass channels were incubated in a $\mathrm{NaOH}$ solution in a plastic Petri dish, while $\mathrm{NaOH}$ was injected in the base inlet of the microchannels. The gelation took $2-9 \mathrm{hrs}$ to complete depending on the length of the channel. The channel was kept still during the gelation, and no change in the $\mathrm{NaOH}$ source was made.

\subsection{Microfabrication}

The $20 \mu \mathrm{m}$ microchannels were fabricated using the standard photo- and soft-lithography protocols. [34, 35] Briefly, a 4 inch silicon wafer was rinsed using acetone and dried, followed by cleaning in a plasma cleaner (PDC-001, Harrick Plasma Inc.) for $30 \mathrm{~s}$ at high intensity. Photoresist SU-8 2025 (MicroChem) at $4000 \mathrm{rpm}$ was spin-coated (WS-650-23 spin coater, Laurell Technologies) on the wafer for $30 \mathrm{~s}$. The wafer was then baked on a hot plate, and exposed to UV light through a transparency photomask with desired patterns (25400 DPI, CAD/Art Services Inc.). The unreacted SU-8 was then dissolved in the SU-8 developer (MicroChem) to form the patterns on the wafer.

To fabricate the microchannels using the prepared silicon master, polydimethylsiloxane (PDMS) resin and curing agent (Sylgard 184, Dow Corning) were mixed at a 10 to 1 ratio, degassed under vacuum, poured on the silicon master and cured for 2 hrs at $70^{\circ} \mathrm{C}$. The $\mathrm{NaOH}$ source and chitosan inlet were then cut from PDMS using 5 and $1.5 \mathrm{~mm}$ biopsy punches, respectively. The prepared PDMS piece was then attached to a coverglass slide (VWR micro slides, $24 \times 40 \mathrm{~mm}$ ) after plasma treatment of both the PDMS and glass surfaces for 2 min at high intensity. 


\subsection{Gold NP synthesis}

Gold NPs were synthesized following Frens protocol. 36 Briefly, $2 \mathrm{ml}$ of a $0.5 \mathrm{wt} \%$ chloroauric acid solution (Sigma-Aldrich) was diluted in $96 \mathrm{ml}$ water

115

(Sigma-Aldrich) was swiftly injected to the solution with vigorous stirring. Upon addition of the trisodium citrate, the color of the solution changed to blue and then gradually became red. This color change is attributed to the increase in size of gold NPs as the citrate ions reduce gold (III). [36] This procedure produces highly spherical monodisperse gold NPs. 37] After cooling the solution in the room temperature, the NPs were PEGylated by mixing $1 \mathrm{ml}$ of a $1 \mathrm{wt} \%$ methoxy-PEG-thiol ( 5 kDa, JenKem Technology) with the NP solution for 8 hrs. The diameter $(d=41 \mathrm{~nm})$ and polydispersity $(\mathrm{PDI}=0.17)$ of the PEGylated gold NPs were measured using dynamic light scattering (Malvern ZetaSizer

125 Nano-ZS). The diameter of the hard core of these NPs (without PEG) was $23 \mathrm{~nm}$.

\subsection{Microscopy techniques}

The phase-contrast images $(2048 \times 2048$ pixels $)$ were taken using an upright bright-field microscope (Olympus BX81) using a 20X magnification phase con-

130 trast objective. The hydrogel samples in microchannels were imaged using this microscope without removing the samples from the microchannel.

The same microscope and objective were used for differential dynamic microscopy (DDM) measurements. The microscope is equipped with a high acquisitionspeed camera (Hamamatsu Orca-Flash 4.0 V3). Videos were recorded at 100adjusted with respect to the dynamics of the nanoparticles to result the best signal-to-noise ratio. The DDM technique and analysis are described in detail in the literature. $38,39,40,41,42,43$,

The DDM experiments on gold NPs in the gels were conducted as follows. After the gelation was completed, the $\mathrm{NaOH}$ was removed from the $5 \mathrm{~mm}$ inlet and replaced by fresh water for $24 \mathrm{hrs}$, while the water was renewed after 1 , 
2, 4 and $18 \mathrm{hrs}$ to ensure a neutral $\mathrm{pH}$ in the gel. The membrane was cut using a biopsy punch. Next, the water was replaced by a concentrated gold NPs solution (1.14 mg ml${ }^{-1}$ ) for $48 \mathrm{hrs,} \mathrm{allowing} \mathrm{the} \mathrm{NPs} \mathrm{diffuse} \mathrm{in} \mathrm{the} \mathrm{gel} \mathrm{by}$ their Brownian motion. To extract the diffusion coefficient in the gel, capillaries and water, separate videos were taken from these regions, and analyses were performed using laboratory-made software. Diffusion of gold NPs in water were measured by selecting ROI in a well within the gel matrix.

Confocal microscopy images were taken using an upright laser scanning confocal microscope (TCS SP2 Leica Microsystems) with a dry 20X objective $(\mathrm{NA}=0.5)$. The samples were excited using a $488 \mathrm{~nm}$ laser, and the signal was obtained due to the fluorescence properties of the chitosan without any dye. Image stacks $(1024 \times 1024$ pixels $\times 1 \mu \mathrm{m}$ per stack $)$ were captured with a frame average of 2 using the LCS software (version 2.6.1, Leica Microsystems).

The microfabricated channels and chitosan hydrogels for cell culture were prepared as explained above under aseptic condition. The PDMS layer was left in ethanol overnight and dried thoroughly before being attached to the glass. After the gelation was completed, the $\mathrm{NaOH}$ was removed from the $5 \mathrm{~mm}$ inlet and replaced by MiliQ water for $24 \mathrm{hrs}$ while the water was renewed after 1, 2, 4 and 18 hours to ensure a neutral $\mathrm{pH}$ in the gel. Next, growth medium (DMEM, Sigma Aldrich, supplied with 10\% foetal bovine serum (FBS), 2 mM HEPES, Sigma Aldrich, $300 \mathrm{U} \mathrm{ml}^{-1}$ penicillin, $300 \mu \mathrm{g} \mathrm{ml}^{-1}$ streptomycin, and $0.75 \mu \mathrm{g}$ $\mathrm{ml}^{-1}$ amphotericin B, Invitrogen) was injected in the inlet for $24 \mathrm{hrs}$.

Bovine ligament fibroblasts were isolated from the metacarpal-phalangeal joint (fetlock joint) of 12-18 months old calves. The ligaments were harvested and rinsed with PBS, minced into $1 \mathrm{~cm}^{3}$ pieces, and digested in DMEM containing $0.25(\mathrm{w} / \mathrm{v} \%)$ collagenase A (Roche Diagnostics Corporation) at $37^{\circ} \mathrm{C}$ in a humidified atmosphere of $5 \% \mathrm{CO}_{2}$ for $36 \mathrm{hrs}$. The viable cells were determined using Trypan Blue dye exclusion assay (Invitrogen) and expanded in growth medium to passage 3 for the subsequent experiments. 
To culture the cells on the hydrogel, the PDMS piece was separated from the glass using a sterilized scalpel. The cells were seeded on the hydrogel at a density of $50 \times 10^{3}$ per $20 \mu \mathrm{l}$ of the growth medium, and kept in an incubator maintained at $37^{\circ} \mathrm{C}, 5 \% \mathrm{CO}_{2}$ and $95 \%$ humidity for $4 \mathrm{hrs}$. Finally, the gel surface was rinsed with growth medium to remove the unattached cells, and the samples were imaged using a Zeiss OxioObserver microscope (Carl Zeiss, Germany).

\subsection{Statistical analysis}

180 chanically stiffer than the rest of the gel. 44] A similar membrane was reported in alginate and collagen hydrogels when the capillaries were formed in these hydrogels. [30, 18 , 
As gelation proceeds, the 1D diffusion of $\mathrm{NaOH}$ in the channel ( $x$ direction in Figure 1 caused the aggregation and transport of chitosan fibers in the direction opposite to the $\mathrm{NaOH}$ diffusion. Therefore, when $\mathrm{NaOH}$ diffused through the pregel solution from both open ends of the channel, a gel-free zone appeared exactly in the middle of the channel as shown in Figure S1B. This suggests that the gel front does not propagate in the $y$ direction, which is important for uniform distribution of capillaries. Nevertheless, capillary propagation was affected on the $z$ direction, as the capillaries curve in the thick channel (see the z-stack images of the capillaries in Figure S1D), and most of them hit the gel boundaries and disappeared before reaching deeper zones in the gel. This can be attributed to the mechanical stress during gelation, caused by deswelling, as the hydrogel bulk deforms. 45] Also, flow turbulence at the $\mathrm{NaOH}$ entrance, caused by the $\mathrm{NaOH}$ injection, may affect the initial orientation of the capillaries. This issue was less pronounced in thinner channels perhaps because a potential flow turbulence close to the entrance transits to laminar flow much faster in microchannels. As shown in Figure S2, decreasing the channel thickness from $1 \mathrm{~mm}$ to $400 \mu \mathrm{m}$, and further down to $20 \mu \mathrm{m}$, promoted straight propagation of the capillaries. In a $20 \mu \mathrm{m}$ microfabricated channel, a single layer of straight capillaries in the hydrogel was spontaneously obtained. Capillaries in this case propagated into the hydrogel chip three times farther than in the thicker channels (shown in Figure 1]. The single layer of capillaries in microchannels was conveniently imaged using a conventional phase-contrast microscope with a high resolution, whereas the capillaries in thick channels had to be imaged using fluorescent confocal microscopes. The orientation of the capillaries from different angles of view in 1000 and $20 \mu \mathrm{m}$ channels is shown in Figure S3 by z-stack confocal images.

225 The membrane in chitosan hydrogels was found to result from rapid aggregation of polymer chains. [19] As will be shown later, in agreement with previous studies of the neutralization kinetics, [46] the rate of gelation in the superficial membrane is at least $\approx 3$ times faster than the regions where the capillaries form. Increasing the $\mathrm{NaOH}$ and chitosan concentrations enhanced the fast aggregation close to the base solution source, causing the thickness of 


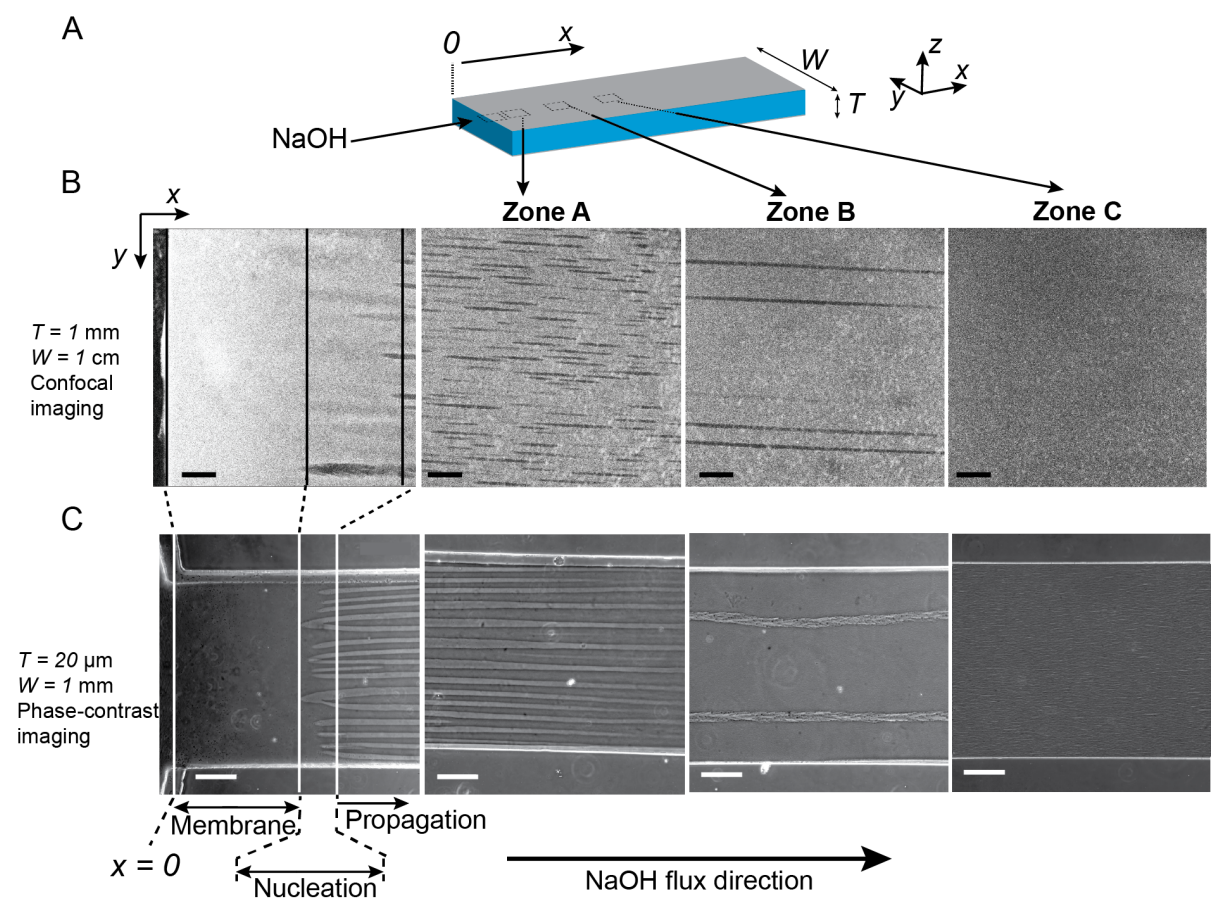

Figure 1: A) Schematic representation of the rectangular channel. Chitosan layer is indicted in blue. B) Fluorescent confocal images illustrating capillary distribution along the $1 \mathrm{~mm}$ thick hydrogel. C) Phase-contrast images of the membrane, nucleation zone and capillary propagation in a $20 \mu \mathrm{m}$ microfabricated hydrogel chip. In these images, the $\mathrm{NaOH}$ concentration is $1 \mathrm{M}$, and chitosan concentration is $1.5 \mathrm{wt} \%$. Scale bars show $100 \mu \mathrm{m}$.

the membrane to increase, as shown in Figure $2 \mathrm{~A}$. Nevertheless, at our optimum gelation conditions, which will be explained to be $1 \mathrm{M} \mathrm{NaOH}$ concentration and $1.5 \mathrm{wt} \%$ chitosan concentration, the membrane thickness, which was found to be independent of the channel thickness, was $\approx 3-10 \%$ of the length of the region with the capillaries, and could be easily cut from the gel if needed. This constitutes a built-in mechanism to produce closed capillaries that can be opened by removing the membrane depending on the application.

The capillaries dimensions were studied with respect to different gelation parameters. For example, the $\mathrm{NaOH}$ concentration was found to greatly im240 pact the capillary formation by changing the concentration gradient. This was 
reported in Figure $2 \mathrm{~B}$ and $2 \mathrm{C}$, at a wide range of base concentration from 0.1-10 M. For all base concentrations, the capillaries diameter was between 10$30 \mu \mathrm{m}$ with a slight increase towards the middle of the gel, and the capillaries occupied less than $20 \%$ of the gel volume. They disappeared far from the $\mathrm{NaOH}$ source (1-4 mm away, depending on the $\mathrm{NaOH}$ source concentration) because of the decreasing gradient of $\mathrm{NaOH}$ concentration from the tip to the middle of the gel. It should be noted that the diffusion of $\mathrm{NaOH}$ in chitosan is also limited by the viscosity of the $\mathrm{NaOH}$ solution, which depends on the base concentration itself. Therefore, by increasing the $\mathrm{NaOH}$ concentration from $0.1 \mathrm{M}$ to $5 \mathrm{M}$, the capillary length and volume fraction increased, whereas by further increasing the concentration to $10 \mathrm{M}$, the $\mathrm{NaOH}$ viscosity significantly hindered the diffusion and capillary formation and propagation (viscosity increases from $2.78 \mathrm{cp}$ for $5 \mathrm{M} \mathrm{NaOH}$ to $12.6 \mathrm{cp}$ for $10 \mathrm{M} \mathrm{NaOH})$.

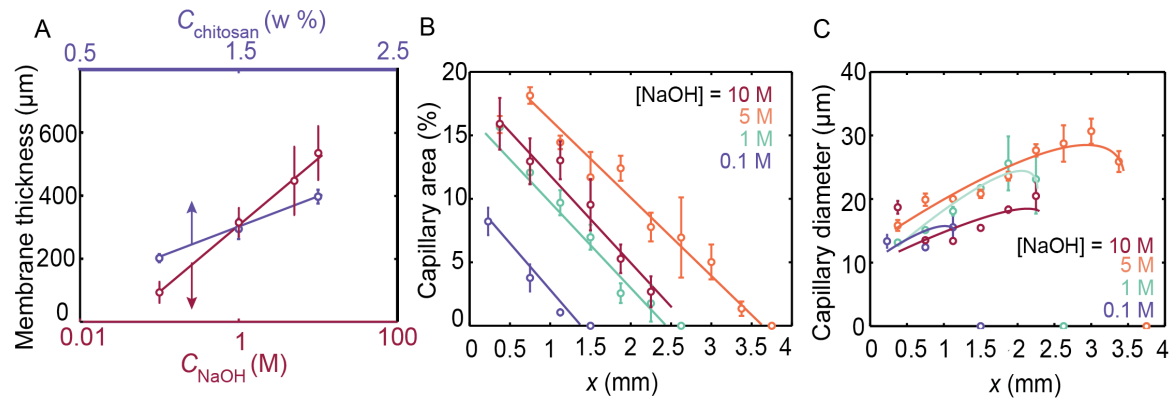

Figure 2: A) The thickness of the membrane increases with chitosan and $\mathrm{NaOH}$ concentrations. B) Percentage of the hydrogel occupied by the capillaries at different base concentrations. C) Variation of the capillary diameter along the hydrogel for different $\mathrm{NaOH}$ concentrations. The analyses here were performed using confocal fluorescent images of several $1 \mathrm{~mm}$-thick hydrogel samples. In panel $\mathrm{A}, C_{\mathrm{NaOH}}=1 \mathrm{M}$ (blue) and $C_{\text {chitosan }}=1.5 \mathrm{wt} \%$ (red). In panels B and C, $C_{\text {chitosan }}=1.5 \mathrm{wt} \%$. Lines are to guide the eyes.

Another important parameter to control the capillary formation is the chitosan concentration. It was found that the capillaries do not form above $2 \mathrm{wt} \%$ chitosan concentration, whereas capillary number density was increased at lower chitosan concentrations (see Figure S4 and S5). However, below $1.5 \mathrm{wt} \%$, the gel was too soft to handle for the subsequent cell culture and NP screening ap- 
plication. Therefore, for practical applications, $1.5 \mathrm{wt} \%$ chitosan concentration appeared to be the optimal condition. At this concentration, the autofluorescence signal from the chitosan was strong enough to eliminate the need for conjugating the polymers with any dye, which facilitated the confocal imaging. Other parameters influencing the multiscale polymer entanglement regimes in chitosan solutions, such as the chitosan molecular weight and degree of acetylation (DA), have been reported to significantly affect the existence of the capillary morphology in the gels. [19] Nevertheless, in the following sections, by using a constant molecular weight $(\mathrm{Mw} \approx 600 \mathrm{kDa}, D=\mathrm{Mw} / \mathrm{Mn}=1.6), \mathrm{DA}=4 \%$, chitosan concentration (1.5 wt\%) and base concentration (=1 M), we focused on engineering hydrogel microdevices with controlled complex capillary patterns 270 by modulating structural parameters.

To this end, the microfabricated channel used for gel preparation was schematically shown in Figure 3A; a tunable parallelepiped gelation microchamber of section $W \times T$ was used and the effect of the microchannel width, $W$, on the capillaries propagation and morphology was investigated. The channel height, $T$, was fixed at $20 \mu \mathrm{m}$ to ensure the presence of a single layer of capillaries in the channel. It was found that the width of the channel, $W$, did not affect the capillary formation as the number of the capillaries per unit width, $N / W$, and their dimensions were independent of $W$, as shown in Figure $3 \mathrm{C}$ and $3 \mathrm{D}$. Nevertheless, the channel height was influential, as the capillary length in the microchannel was approximately three times that in the $1 \mathrm{~mm}$-thick channel as shown in Figure $2 \mathrm{~B}$ and $3 \mathrm{C}(\approx 8$ vs. $\approx 2.5 \mathrm{~mm})$. Therefore, a better control over the 1D diffusion in the channel significantly improves the capillary propagation. The diameter of the capillaries in the microchannel, on the other hand, remained unchanged, ranging from 10-30 $\mu \mathrm{m}$, similarly to the thick channels.

285 Excluding the membrane, three different regions were observed in the gels. In the first region, zone $\mathrm{A}$, capillaries were unstable, i.e., they propagated at a high rate of $v>2 \mu \mathrm{m} \mathrm{s}^{-1}$ (see Figure $3 \mathrm{~B}$ ), but disappeared deeper inside the gel. The number of these capillaries decreased exponentially with propagation distance, $x$, and their interior was uniformly hollow according to the phase- 
A

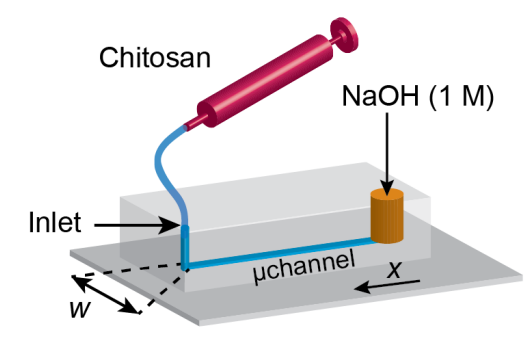

C

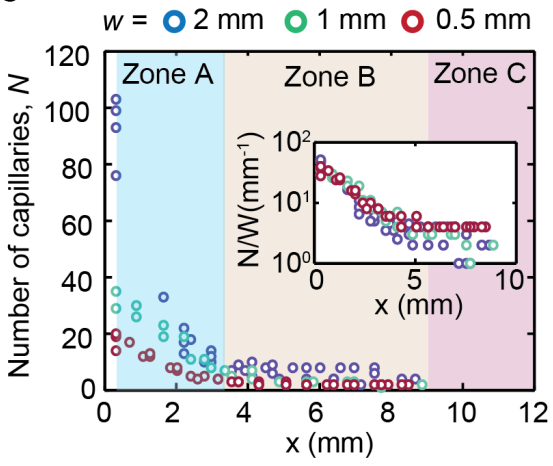

B

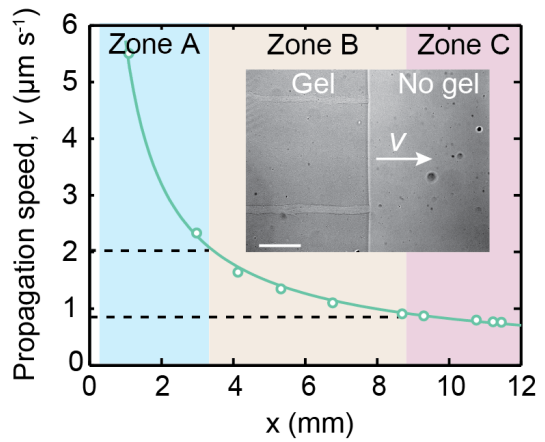

D

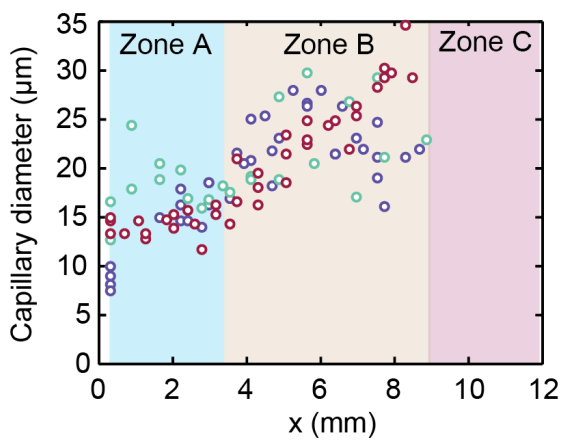

Figure 3: A) Schematic representation of the hydrogel chip fabrication setup. B) Gelation propagation speed at different gelation zones. The inset shows an image of the gelation front propagation in zone B. The solid line shows the best fit $v=5.72 x^{-0.842}$. C) Variation of the number of capillaries $N$ along the hydrogel chip. The inset shows that the scaled number of capillaries with the channel width forms a master curve. D) Variation of the capillary diameter along the hydrogel microlayer.

contrast images (see Figure 1C). Despite their lower number density, capillaries that reached zone $\mathrm{B}(x \gtrsim 3.5 \mathrm{~mm})$ were significantly more stable. Their number density and diameter were almost constant, and they were easily reproducible, unlike the capillaries in zone A that deformed under geometrical imperfections in the channel, as will be discussed below. The gelation propagation speed in zone $\mathrm{B}$ was between 1 to $2 \mu \mathrm{m} \mathrm{s}^{-1}$. The internal structure of capillaries in zone B seemed to be nonuniform, suggesting that there might be polymer residues remaining in the capillaries, as shown in the phase-contrast images (see Figure $1 \mathrm{C}$ ). However, by monitoring the diffusion of NPs in the capillaries, it 
will be demonstrated below that the capillaries were completely hollow. The roughness. Zone $\mathrm{C}(x \gtrsim 9 \mathrm{~mm})$ was the region without any capillaries. It contained gel with larger pores as can be seen in the phase-contrast images (also reported by [19]). The gelation propagation speed was slow in this region $\left(v<1 \mu \mathrm{m} \mathrm{s}^{-1}\right)$. It was mentioned that the membrane at the interface of 305 rest of the gel $\left(v>15 \mu \mathrm{m} \mathrm{s}^{-1}\right)$. Therefore, for the capillaries to form, the gelation propagation speed must be between a certain limit $(1<v<15 \mu \mathrm{m}$ $\left.\mathrm{s}^{-1}\right)$. This is consistent with the proposed mechanism for capillary formation based on the friction between the contracting polymer chains and surrounding liquid, 20, 21, 22] since the friction force depends on the coagulation speed.

So far, the experiments were performed in straight microchannels, which allowed producing straight capillaries with known number densities. Spatial distribution and shape of these capillaries can be controlled by tuning the channel geometry. For example, introducing micropillars in the channel creates wavy microcapillaries, more predominantly in zone B, while in zone A they tend to be unstable (see Figure 4). Instability appeared as fingering patterns in the postobstacle regions and depended on the capillary number density. The minimum distance from the obstacle for the capillaries to avoid fingering because of the post-obstacle instabilities $d_{\min }=38.6 \pm 1.3 \mu \mathrm{m}$ (independent of the obstacles size or shape). If the inter-capillary distance $d>d_{\min }$, the capillaries were able to bypass the obstacle without fingering, adopting a wavy shape. This was the case in zone $\mathrm{B}$, where the capillary density was low (typically $N / W<10 \mathrm{~mm}^{-1}$ ). However, the high capillary density in zone A (typically $N / W>20 \mathrm{~mm}^{-1}$ ) led to $d<d_{\min }$, pushing the capillaries towards the post-obstacle region with 325 flow instabilities. Noteworthy is that these instabilities in zone A were resolved along the gel and capillary propagation was continued to zone B and eventually stopped at zone C. Different micropillar densities and geometries were shown in Figure S6, having similar effect on the propagation of the capillaries in zones A and B. The complex flow-pattern strategy may be used to create other complex 
capillary patterns. For example, by changing $\mathrm{NaOH}$ source geometry, complex flow patterns can be generated at the entrance of the channel, affecting the orientation of the capillaries or inducing branching (see Figure S7).
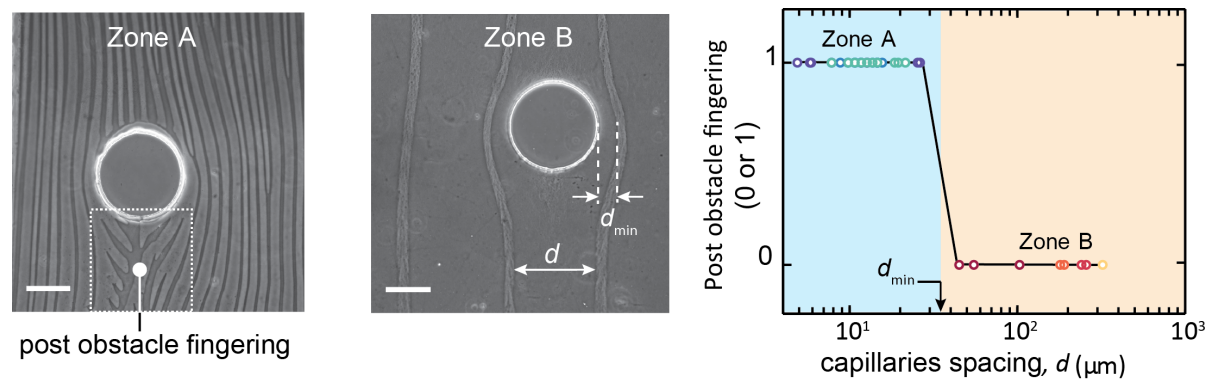

Figure 4: Post obstacle fingering in different capillary formation zones. Capillary fingering is represented by 1 , and lack of post-obstacle fingering is translated to 0 . The phase-contrast images show capillary's post-obstacle behavior in zone $\mathrm{A}$, where $d<d_{\mathrm{min}}$, and zone $\mathrm{B}$, where $d>d_{\text {min }}$. Scale bars show $100 \mu \mathrm{m}$. See Figure $\mathrm{S} 6$ for more images with different obstacles.

As introduction of obstacles in the channel can distort the capillaries, manipulating the $\mathrm{NaOH}$ diffusion flux in non-straight channels can promote a similar effect. Controlled capillary branching can be induced in zone B by changing the diffusion pathway locally as shown in Figure 5. For example, channels expansion with different expansion angles $\theta$, creates a complex, yet highly defined, pattern of branched capillaries (Figure 5A). Figure 5B shows that with increasing the expansion angle $\theta$, more capillary branching occurs, with a minimum threshold of $\theta \sim 14^{\circ}$, below which the deformation rate was insufficient to create such an impact. However, some of the small capillary branches disappeared after travelling over a few millimeters.

Capillary branching could also occur by an abrupt change of channel direction (channel elbow). In this geometry, branching was systematically obtained in the region passed and near the channel elbow, and was tuned by changing the elbow angle, as shown in Figure 5C. It was found that the branching distance from the elbow tip $L_{f}$, was inversely proportional to the elbow angle $\theta$ (Figure 5D). The capillary branching can be attributed to diffusive flow displacement instability upon the sudden transition from one-dimensional to 
bending, as well as in the region immediately after obstacles. A detailed theoretical approach, coupling the hydrodynamic and diffusion-reaction models in this condition is proposed as a future study to elucidate the specific regime at which the instabilities emerge. Finally, as shown in Figure $5 \mathrm{E}$, the capillaries to the straight channels. The length of the capillaries did not appear to depend on $R$ (when $R>2 \mathrm{~mm}$ ), and no branching was observed (Figure $5 \mathrm{~F}$ ). The reproducibility of various capillary patterns formed in different channel geometries can be advantageous in generating biomimetic "microvascularized" soft matrices for recapitulating tissue microenvironments, or hydrogel patches capable of trapping and releasing nanomaterials with applications in nanomedicine or NP-assisted imaging.

To demonstrate the potential application of these microvascularized hydrogels, we first used the microcapillaries as loading vessels for gold NPs. The presence of capillaries in the chitosan hydrogels produces a microstructure that provides embedded NPs with multiple diffusion patterns in the membrane, capillaries and the gel matrix.

As mentioned earlier, the dense membrane can be used as a built-in mechanism to close or open the capillaries. To test this property, we produced a hydrogel disc with a diameter of $9 \mathrm{~mm}$, whose capillaries were converging radially towards the center of the disc (see Figure S8A). In this configuration, the membrane covered the sides of the disc and closed the capillaries (the faces of the discs were covered during $\mathrm{NaOH}$ diffusion). Upon completion of the gelation, the $\mathrm{NaOH}$ in the disc was removed by soaking the gel in DI water. When only the side of the disc was exposed to PEGylated colloidal gold NPs of $\approx 41 \mathrm{~nm}$ diameter, the radial diffusion of NPs was hindered by the membrane within 48 hrs. This suggests that the membrane has a homogeneous dense structure with pores smaller than the NP size (see Figure S8B and C). However, after removing the membrane using a biopsy punch and re-exposing the sides of the 380 disc to the same NPs, they spread in the gel in less than 24 hrs by diffusion 

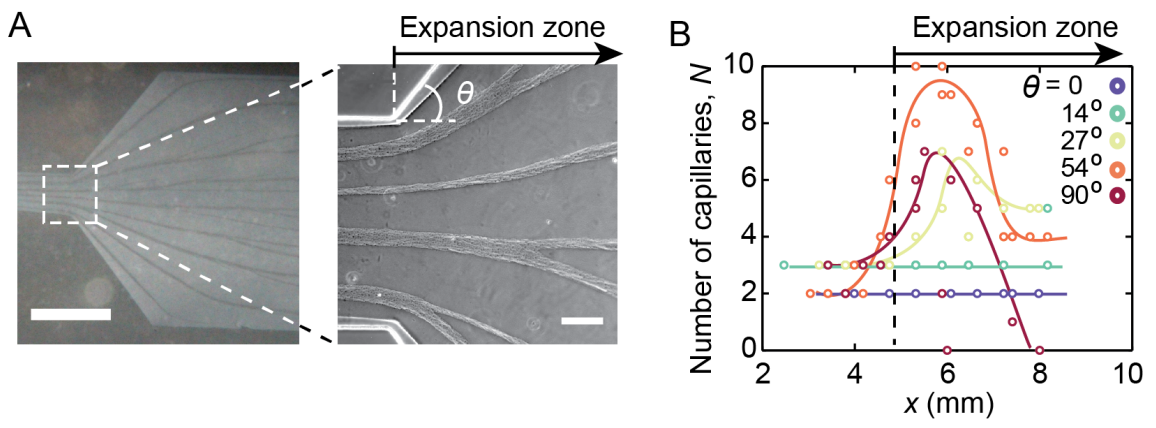

C
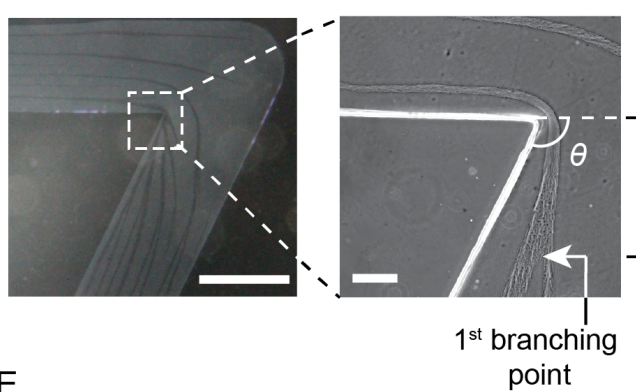

$\mathrm{D}$

E
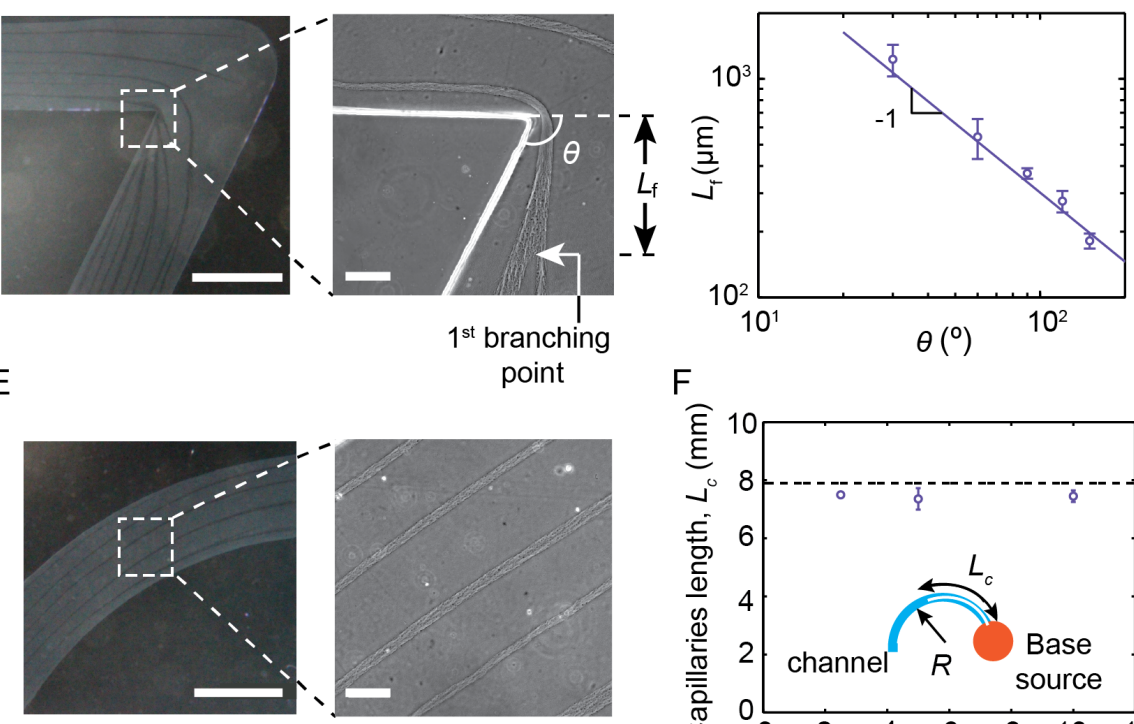

$\mathrm{F}$

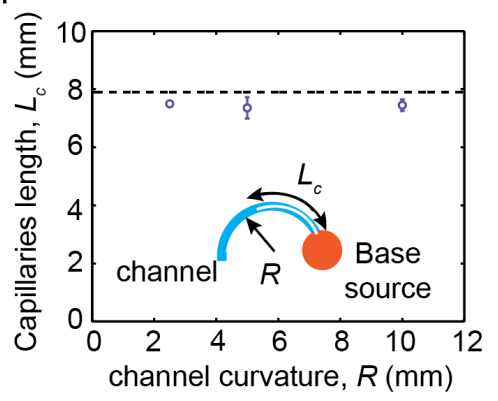

Figure 5: A) Phase-contrast images of the capillary branching during channel expansion with expansion angle $\theta$. B) Variation of the number of capillaries along zone B, where capillaries were stable. Data points on each curve were obtained from multiple samples. C) Phasecontrast images of capillary branching because of a channel elbow. D) The position of the first branching point from the elbow, $L_{f}$, versus the bending angle $\theta$. The solid line correspond to $L_{f} \approx 3.8 \times 10^{4} \theta^{-1.0}$. E) Phase-contrast images of capillaries in a curve channel of curvature radius $R$. F) The dependence of the capillary length, $L_{c}$, to the channel curvature. The dashed line shows the length of the capillaries in a straight channel of equal dimensions. Scale bars in zoomed-out images correspond to $1 \mathrm{~mm}$ and in zoomed-in images show $100 \mu \mathrm{m}$. 
through the capillaries (see Figure S8D and E). These capillaries could potentially be closed again by depositing a thin layer of pregel solution around the sides of the disc and exposing the solution to $\mathrm{NaOH}$.

We also used these structured matrices to mimic microvascularized tissues and characterize the transport of NPs in such complex environment. Microfluidic platforms that enable studying nanomedicines navigation in microvessels and their escape from the blood stream to reach their targeted tissue and cells are critically missing. The present hydrogel fabrication approach provides the means to build a such microdevice platform and to adapt its configuration to many types of microvascularized tissues. Moreover, chitosan hydrogels in form of macroscopic, small diameter vascular substitutes were previously internally covered by endothelial progenitor cells, suggesting that chitosan hydrogels possess the surface and biomaterial properties suitable for such biofunctionalization [7].

We built a vascularized hydrogel matrix with microwells occupied with fibroblasts cells. The microwells were introduced in the hydrogel chip by embedding pillars in the channel during the fabrication of the microchannel using photolithography. Then, the microchannel was filled with chitosan solution, and capillaries were generated by self assembly during the gelation process (see Figure 6A and B). The hydrogel was then rinsed in DI water and soaked in culture medium. Finally, the gelation chamber was opened and the cells were seeded in the wells (see Figure S9). We used this tunable complex hydrogel microenvironment to characterize the different diffusion behaviors that gold NPs adopt when traveling in complex environments. The present model mimics a biological tissue, where nanomedicine has to travel through the vasculature, extravasate into and diffuse through the extracellular matrix and eventually reach targeted cells.

The local diffusion coefficients of gold NPs in capillaries and the gel were measured by DDM, 38, 48, 49] which is a correlation-based microscopy technique capable of measuring the diffusion coefficient in complex viscoelastic media with high precision without the necessity to perform any particle tracking. 
A

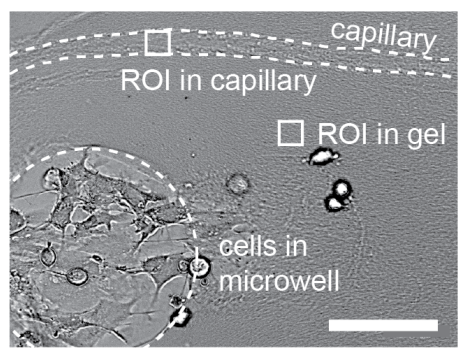

C

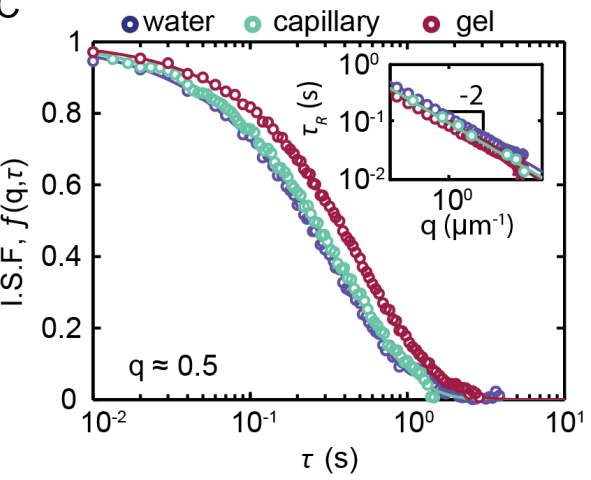

B

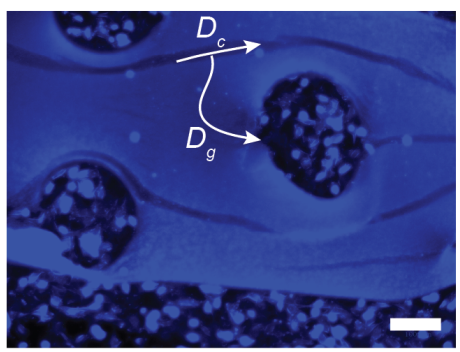

D

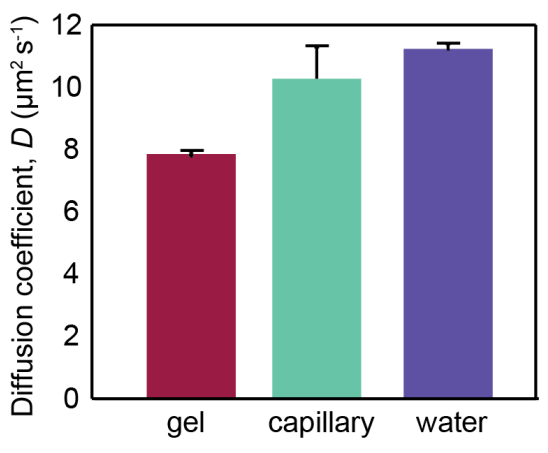

Figure 6: A) Fibroblast cells cultured in $200 \mu \mathrm{m}$-wide cylindrical wells distributed in chitosan hydrogel with capillaries. The dashed lines are guides to the eyes and delineate the edges of a single capillary and well. Also indicated, the different regions of interest (ROIs) used in DDM to measure locally the diffusion coefficient of gold NPs. The diffusion coefficient in water was measured by choosing the ROI in the microwells without the cells. B) A widen view of the cultured cells under blue filter. The overall NP diffusion directions in the capillary, $D_{c}$, and gel, $D_{g}$, are also shown by arrows upon introduction to the channel. C) Representations of the measured intermediate scattering function $f(q, \tau)=\exp -\left(\tau / \tau_{R}\right)$ versus the delay time for gold NPs in gel, capillaries and water. The inset confirms that the measured relaxation times scale with the wavenumber $q$ as $\tau_{R} \sim q^{-2}$, as expected for a diffusive motion. D) The extracted diffusion coefficients, $D$, of $\approx 41 \mathrm{~nm}$ gold NPs from the ISF. The values of $D$ were averaged over 5 freshly prepared samples and a wide range of scattering wavevector values $(q=0.3-2.4)$. Scale bars show $100 \mu \mathrm{m}$.

DDM yields the $q^{\text {th }}$ Fourier component of the temporal autocorrelation function of the particle density, $f(q, \tau)$, also called the Intermediate Scattering Function (ISF), with $\tau$ the delay time. By performing DDM at different positions in

the hydrogel, it was found that the ISF can be approximately described by an 
exponential function (see Figure $6 \mathrm{C}$ ), as expected for non-interacting Brownian particles, with the relaxation time $\tau_{R}=1 /\left(q^{2} D\right)$ (see Figure $6 \mathrm{C}$ inset), and $D$ the diffusion coefficient. The values of the diffusion coefficient obtained in capillaries and the hydrogel bulk were compared to the diffusion coefficient of gold NPs in water (in microwells without cells, Figure 6D) using statistical analysis. The results suggested that there were a statistically significant difference between the diffusion coefficient in these three media (ANOVA, $p=0.0012$ ). The pair-wise comparison between the diffusion coefficients in these media showed that the gold NPs diffuse in capillaries almost as fast as in water (Tukey-Kramer, $p=0.20$ ), while in smaller pores of the gel their diffusion was slowed by the gel matrix significantly (Tukey-Kramer, $p=0.0062$ for gel versus capillary, and $p=0.0011$ for gel versus water) similarly to the diffusion of NPs in natural tissues. [50, 51, 52. The pore size of the $1.5 \mathrm{wt} \%$ chitosan hydrogel was estimated around $40-80 \mathrm{~nm}$ according to the measured diffusivity of probe particles with different sizes. 53

The elastic modulus of the chitosan hydrogel used in this study (considering the concentration, DA and molecular weight of the chitosan) is estimated to be $\approx 1 \mathrm{kPa}$ according to rheological studies [28]. This elastic modulus is comparable to the elastic moduli of soft tissues, such as healthy breast tissues ${ }_{435}(0.4-2 \mathrm{kPa})$ [54, spleen $(1-10 \mathrm{kPa})$ [55, 56, liver $(0.4-1.0 \mathrm{kPa})$ [57] and muscle $(1.2-3.2 \mathrm{kPa})$ [57]. The diameter of the self-assembled capillaries $(10-30 \mu \mathrm{m})$ is also comparable to the typical diameter of arterioles $(5-100 \mu \mathrm{m})$, metarterioles (10-20 $\mu \mathrm{m})$ and the upper limit of blood capillaries $(5-10 \mu \mathrm{m})$ [58]. The multiple diffusive patterns in vascularized hydrogel, combined with the level of control on the capillary patterning in the chitosan hydrogel chips, suggest that a network of branched and deformed self-assembled capillaries can effectively mimic microcirculation network of various tissues, and the diffusion of nanomaterials to the cells that are embedded in tissue microenvironment. 


\section{Conclusion}

445 terned hydrogels in a microfluidic setup. A simple single-step procedure for the preparation of capillaries and lack of any toxic residues distinguish this technique from other hydrogel microengineering techniques. Quantitative analyses showed that the capillaries in the microchannels can be deformed and branched readily under the influence of the gelation agent diffusion by changing the channel geometry, diffusion rate or diffusion pathway. The microvascularized gel was successfully tested as a media with multiple diffusion patterns that can be potentially used to mimic a vascularized tissue for drug delivery to cells embedded within the tissue. In addition, the detailed analyses of the capillary propagation and deformation in the microfluidic channel presented in this study provide novel opportunities for theoretical studies to further investigate the effective parameters that can facilitate hydrogel patterning using these self-assembled structures.

\section{Acknowledgements}

Financial support from NSERC and CRC is gratefully acknowledged (XB and DKH). VA was supported by FRQNT postdoctoral research scholarship. This research was undertaken thanks, in part, to funding from the Canada First Research Excellence Fund through the TransMedTech Institute. 


\section{References}

[1] K. Y. Lee, D. J. Mooney, Hydrogels for tissue engineering, Chem. Rev. 101 (2001) 1869-1879.

[2] A. Khademhosseini, R. Langer, Microengineered hydrogels for tissue engineering, Biomaterials 28 (2007) 5087-5092.

[3] J. Thiele, Y. Ma, S. M. C. Bruekers, S. Ma, W. T. S. Huck, 25th anniversary article: designer hydrogels for cell cultures: a materials selection guide, Adv. Mater. 26 (2014) 125-148.

[4] S. R. Caliari, J. A. Burdick, A practical guide to hydrogels for cell culture, Nat. Methods 13 (2016) 405-414.

[5] J. Li, D. J. Mooney, Designing hydrogels for controlled drug delivery, Nat. Rev. Mater. 1 (2016) 16071.

[6] T. R. Hoare, D. S. Kohane, Hydrogels in drug delivery: progress and challenges, Polymer 49 (2008) 1993-2007.

[7] V. Adibnia, R. J. Hill, Universal aspects of hydrogel gelation kinetics, percolation and viscoelasticity from pa-hydrogel rheology, J. Rheol. 60 (2016) $541-548$.

[8] H. Geckil, F. Xu, X. Zhang, S. Moon, U. Demirci, Engineering hydrogels as extracellular matrix mimics, Nanomedicine 5 (2010) 469-484.

[9] S. Khetan, J. A. Burdick, Patterning hydrogels in three dimensions towards controlling cellular interactions, Soft Matter 7 (2011) 830-838.

[10] Y. Cheng, Y. Yu, F. Fu, J. Wang, L. Shang, Z. Gu, Y. Zhao, Controlled fabrication of bioactive microfibers for creating tissue constructs using microfluidic techniques, ACS Appl. Mater. Interfaces.

[11] R. A. Barry III, R. F. Shepherd, J. N. Hanson, R. G. Nuzzo, P. Wiltzius, J. A. Lewis, Direct-write assembly of 3d hydrogel scaffolds for guided cell growth, Adv. Mater. 21 (2009) 2407-2410. 
[12] J. W. Nichol, S. T. Koshy, H. Bae, C. M. Hwang, S. Yamanlar, A. Khademhosseini, Cell-laden microengineered gelatin methacrylate hydrogels, Biomaterials 31 (2010) 5536-5544.

[13] K. Yue, G. Trujillo-de Santiago, M. M. Alvarez, A. Tamayol, N. Annabi, A. Khademhosseini, Synthesis, properties, and biomedical applications of gelatin methacryloyl (gelma) hydrogels, Biomaterials 73 (2015) 254-271.

[14] J. Wang, J. Chao, H. Liu, S. Su, L. Wang, W. Huang, I. Willner, C. Fan, Clamped hybridization chain reactions for the self-assembly of patterned dna hydrogels, Angew. Chem. Int. Ed. 129 (2017) 2203-2207.

[15] C. Huang, D. Quinn, S. Suresh, K. J. Hsiaa, Controlled molecular selfassembly of complex three-dimensional structures in soft materials, Proc. Natl. Acad. Sci. 115 (2018) 70-74.

[16] H. Wang, A. Paul, D. Nguyen, A. Enejder, S. C. Heilshorn, Tunable control of hydrogel microstructure by kinetic competition between self-assembly and crosslinking of elastin-like proteins, ACS Appl. Mater. Interfaces 10 (2018) 21808-21815.

[17] E. Schuster, J. Eckardt, A. Hermansson, A. Larsson, N. Lorén, A. Altskar, A. Strom, Microstructural, mechanical and mass transport properties of isotropic and capillary alginate gels, Soft Mater 10 (2014) 357-366.

[18] K. Furusawa, S. Sato, J. Masumoto, Y. Hanazaki, Y. Maki, T. Dobashi, T. Yamamoto, A. Fukui, N. Sasaki, Studies on the formation mechanism and the structure of the anisotropic collagen gel prepared by dialysisinduced anisotropic gelation, Biomacromolecules 13 (2012) 29-39.

[19] N. Sereni, A. Enache, G. Sudre, A. Montembault, C. Rochas, P. Durand, M. Perrard, G. Bozga, J. Puaux, T. Delair, 1. David, Dynamic structuration of physical chitosan hydrogels, Langmuir 33 (2018) 12697-12707.

[20] J. Thumbs, H. H. Kohler, Capillaries in alginate gel as an example of dissipative structure formation, Chem. Phys. 208 (1996) 9-24. 
[21] H. Treml, H. H. Kohler, Coupling of diffusion and reaction in the process of capillary formation in alginate gel, Chem. Phys. 252 (2000) 199-208.

[22] H. Treml, S. Woelki, H. H. Kohler, Theory of capillary formation in alginate gels, Chem. Phys. 293 (2003) 341-353.

[23] A. Onuki, S. Puri, Spinodal decomposition in gels, Phys. Rev. E 59 (1999) R1331.

525 [24] J. E. Maneval, D. Bernin, H. T. Fabich, J. D. Seymour, S. L. Codd, Magnetic resonance analysis of capillary formation reaction front dynamics in alginate gels, Magn. Reson. Chem. 49 (2011) 627-640.

[25] T. Kean, M. Thanou, Biodegradation, biodistribution and toxicity of chitosan, Adv. Drug Deliv. Rev. 62 (2010) 3-11.

[26] M. W. Weinhold, J. Sauvageau, J. Kumirska, J. Thoming, Studies on acetylation patterns of different chitosan preparations, Carbohydr. Polym. 78 (2009) 678-684.

[27] S. Popa-Nita, P. Alcouffe, C. Rochas, L. David, A. Domard, Continuum of structural organization from chitosan solutions to derived physical forms, Biomacromolecules 11 (2010) 6-12.

[28] A. Montembault, C. Viton, A. Domard, Rheometric study of the gelation of chitosan in aqueous solution without cross-linking agent, Biomacromolecules 6 (2005) 653-662.

[29] J. Faivre, G. Sudre, A. Montembault, S. Benayoun, X. Banquy, T. Delair, L. David, Bioinspired microstructures of chitosan hydrogel provide enhanced wear protection, Soft Matter 14 (2018) 2068-2076.

[30] P. Prang, R. Muller, A. Eljaouhari, K. Heckmann, W. Kunz, T. Weber, C. Faberc, M. Vroemen, U. Bogdahn, N. Weidner, The promotion of oriented axonal regrowth in the injured spinal cord by alginate-based anisotropic capillary hydrogels, Biomaterials 27 (2006) 3560-3569. 
[31] W. A. Anderson, A. R. Willenberg, A. J. Bosak, B. J. Willenberg, S. Lambert, Use of a capillary alginate gel (capgel $\left.{ }^{\mathrm{tm}}\right)$ to study the threedimensional development of sensory nerves reveals the formation of a rudimentary perineurium, J. Neurosci. Methods 305 (2018) 46-53.

[32] S. Barua, S. Mitragotri, Challenges associated with penetration of nanoparticles across cell and tissue barriers: A review of current status and future prospects, Nano Today 9 (2014) 223-243.

[33] A. Hirai, H. Odani, A. Nakajima, Determination of degree of deacetylation of chitosanby h nmr spectroscopy, Polym. Bull. 26 (1991) 87-94.

[34] Y. Xia, G. M. Whitesides, Soft lithography, Angew. Chem. Int. Ed. 37 (1998) 550-575.

[35] B.-U. Moon, S. G. Jones, D. K. Hwang, S. S. H. Tsai, Microfluidic generation of aqueous two-phase system (atps) droplets by controlled pulsating inlet pressures, Lab. Chip. 15 (2015) 2437-2444.

[36] G. Frens, Controlled nucleation for the regulation of the particle size in monodisperse gold suspensions, Nature Phys. Sci. 241 (1973) 20-22.

[37] S. Kumar, J. Aaron, K. Sokolov, Directional conjugation of antibodies to nanoparticles for synthesis of multiplexed optical contrast agents with both delivery and targeting moieties, Nat. Protoc. 3 (2008) 314-320.

[38] R. Carbino, V. Trappe, Differential dynamic microscopy: Probing wave vector dependent dynamics with a microscope, Phys. Rev. Lett. 100 (2008) 188102.

[39] F. Giavazzi, D. Brogioli, V. Trappe, T. Bellini, R. Cerbino, Scattering information obtained by optical microscopy: Differential dynamic microscopy and beyond, Phys. Rev. E. 80 (2009) 031403.

[40] P. J. Lu, F. Giavazzi, T. E. Angelini, E. Zaccarelli, F. Jargstorff, A. B. Schofield, J. N. Wilking, M. B. Romanowsky, D. A. Weitz, R. Cerbino, 
Characterizing concentrated, multiply scattering, and actively driven fluorescent systems with confocal differential dynamic microscopy, Phys. Rev. Lett. 108 (2012) 218103.

[41] M. Reufer, V. A. Martinez, P. Schurtenberger, W. C. K. Poon, Differential dynamic microscopy for anisotropic colloidal dynamics, Langmuir 28 (2012) $4618-4624$.

[42] K. He, M. Spannuth, J. C. Conrad, R. Krishnamoorti, Diffusive dynamics of nanoparticles in aqueous dispersions, Soft Matter 8 (2012) 11933-11938.

[43] A. V. Bayles, T. M. Squires, M. E. Helgeson, Dark-field differential dynamic microscopy, Soft Matter 12 (2016) 2440-2452.

[44] A. Fiamingo, A. Montembault, S.-E. Boitard, H. Naemetalla, O. Agbulut, T. Delair, S. P. Campana-Filho, P. Menasche, L. David, Chitosan hydrogels for the regeneration of infarcted myocardium: preparation, physicochemical characterization, and biological evaluation, Biomacromolecules 17 (2016) $1662-1672$.

[45] X. Qu, A. Wirsen, A.-C. Albertsson, Novel ph-sensitive chitosan hydrogels: Swelling behavior and states of water, Polymer 41 (2000) 4589-4598.

[46] A. A. Enache, L. David, J.-P. Puaux, I. Banu, G. Bozga, Kinetics of chitosan coagulation from aqueous solutions, J. Appl. Polym. Sci. 135 (2018) 46062 .

[47] A. Aussel, N. B. Thebaud, X. Berard, V. Brizzi, S. Delmond, R. Bareille, R. Siadous, C. James, J. Ripoche, M. Durand, A. Montembault, B. Burdin, D. Letourneur, N. L'Heureux, L. David, L. Bordenave, Chitosan-based hydrogels for developing a small-diameter vascular graft: in vitro and in vivo evaluation, Biomed. Mater. 12 (2017) 065003.

[48] V. A. Martinez, R. Besseling, O. A. Croze, J. Tailleur, M. Reufer, J. Schwarz-Linek, L. G. Wilson, M. A. Bees, W. C. K. Poon, Differen- 
tial dynamic microscopy: A high-throughput method for characterizing the motility of microorganisms, Biophys. J. 103 (2012) 1637-1647.

[49] L. G. Wilson, V. A. Martinez, J. Schwarz-Linek, J. Tailleur, G. Bryant, P. N. Pusey, W. C. K. Poon, Differential dynamic microscopy of bacterial motility, Phys. Rev. Lett. 106 (2011) 018101.

[50] R. G. Thorne, C. Nicholson, In vivo diffusion analysis with quantum dots and dextrans predicts the width of brain extracellular space, Proc. Natl. Soc. Sci. 103 (2006) 5567-5572.

[51] C. Wonga, T. Stylianopoulos, J. Cui, J. Martin, V. P. Chauhan, W. Jiang, Z. Popovic, R. K. Jain, M. G. Bawendi, D. Fukumura, Multistage nanoparticle delivery system for deep penetration into tumor tissue, Proc. Natl. Soc. Sci. 108 (2011) 2426-2431.

[52] V. Raeesi, C. W. Chan, Improving nanoparticle diffusion through tumor collagen matrix by photo-thermal gold nanorods, Nanoscale 8 (2016) $12524-12530$.

[53] J. Faivre, G. Sudre, A. Montembault, S. Benayoun, X. Banquy, T. Delair, L. David, Bioinspired microstructures of chitosan hydrogel provide enhanced wear protection, Soft Matter.

[54] A. Khavari, M. Nyden, D. A. Weitz, A. J. Ehrlicher, Composite alginate gels for tunable cellular microenvironment mechanics, Sci. Rep. 6 (2016) 30854 .

[55] K. Arda, N. Ciledag, E. Aktas, B. K. Aribas, K. Kose, Quantitative assessment of normal soft-tissue elasticity using shear-wave ultrasound elastography, AJR Am. J. Reontgenol. 197 (2011) 532-536.

[56] J. Liu, H. Zheng, P. S. P. Poh, H. Machens, A. F. Schilling, Hydrogels for engineering of perfusable vascular networks, Int. J. Mol. Sci. 16 (2015) $15997-16016$. 
[57] E. J. Chen, J. Novakofski, W. K. Jenkins, W. D. J. OBrien, Youngs modulus measurements of soft tissues with application to elasticity imaging, IEEE Trans. Ultrason. Ferroelectr. Freq. Control. 43 (1996) 191-194.

${ }_{630}$ [58] A. J. Pappano, W. G. Wier, Cardiovascular Physiology, 10th Edition, 2013, Ch. 8 - The Microcirculation and Lymphatics, pp. 153-170. 